\title{
LEOPARD syndrome: a case report and literature review
}

\author{
Authors: Morteza Moatamedi ${ }^{A}$ and Mohammad Derakhshan ${ }^{B}$
}

\section{Clinical findings}

A 36-year-old male presented to the dermatology department with multiple pigmented macular lesions on his skin present for most of his life. Multiple freckles were noted on non-light exposed areas, mainly on his trunk; light exposed areas such as his face were relatively spared. There were no associated symptoms and he was not on any medication. His mother had similar lesions. Biopsy results from a fleshy lesion on his back reported a simple lentigo. He was initially diagnosed as having 'generalised lentiginosis'. Meanwhile he had a lesion excised from his chin that reported intradermal naevus. Patient was reassured.

He was referred again 3 years later with a lesion on his back; excisional biopsy favoured a benign intra-dermal naevus. Absence of underlying medical conditions as well as a lack of associated symptoms suggested the same diagnosis 'lentiginosis profusa'; however, on consultation with clinical geneticists, the presence of signs of hypertelorism (wideset ears) with striking blue eyes and low-set posterior rotated ears was emphasised. These clinical features along with multiple lentigines confirmed a diagnosis of LEOPARD syndrome. While the patient had no genital abnormality, he had stable mild cardiomyopathy. The patient had no short stature, bleeding disorders or developmental abnormalities characteristic of Noonan syndrome.

\section{Discussion}

LEOPARD is an acronym for: lentigines, electrocardiographic conduction defects, ocular hypertelorism, pulmonary valve stenosis, abnormalities of genitalia, retardation of growth and deafness. LEOPARD is multiple congenital anomaly syndrome inherited in an autosomal dominant manner with full penetrance and variable expressivity. ${ }^{1}$ Clinical diagnosis is based on multiple lentigines, typical facial features and cardiac anomalies. It was first reported by Zeisler and Becker in 1936, in a female patient presenting with multiple lentigines (increasing in number from birth to puberty), pectus carinatum, hypertelorism and prognathism. ${ }^{2}$ Around 200 patients have been reported worldwide.

The lentigines may be congenital, but commonly manifest by the age of five and increase throughout puberty. Ocular hypertelorism, palpebral ptosis and low-set ears are common facial characteristics. Stature is usually below the 25 th centile. Cardiac defects, in particular hypertrophic cardiomyopathy mostly involving the left ventricle, and electrocardiogram (ECG) anomalies are common. Additional common features are café-au-lait spots, chest anomalies, cryptorchidism, delayed puberty, hypotonia, mild developmental delay, sensorineural deafness and learning difficulties. $^{3}$

The main genetic abnormality is associated with tyrosine phosphatase SHP2 (PTPN11) gene mutation. This gene is located on chromosome 12q24.1. Germline mutations in PTPN11 cause Noonan and LEOPARD syndromes, which have overlapping clinical features. ${ }^{4}$

While the lentigines remain an aesthetic problem, hypertrophic cardiomyopathy needs careful risk assessment and prophylaxis against sudden death in patients at risk.

\section{Conflict of interest statement}

None declared.

\section{References}

1 Kim J, Kim MR, Kim H], Lee KA, Lee MG. LEOPARD syndrome with PTPN11 gene mutation showing six cardinal symptoms of LEOPARD. Ann Dermatol 2011;23:232-5.

2 Zeisler EP, Becker WS. Generalised lentigo. Arch Dermat Syph 1963:33:109-25.

3 Sarkozy A, Digilio MC, Dallapiccola B. Leopard syndrome. Orphanet J Rare Dis 2008;3:13.

4 Stewart RA, Sanda T, Widlund HR et al. Phosphatase-dependent and -independent functions of Shp2 in neural crest cells underlie LEOPARD syndrome pathogenesis. Dev Cell 2010;18:750-62.

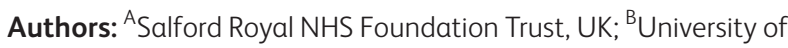
Glasgow, UK 